\section{Comment on "Persistent Currents in Mesoscopic Rings by Suppression of Charge Fluctuations"}

In Ref. [1] and the following two [2,3] Letters, an analytic expression is derived for the average flux-dependent equilibrium current at $T=0$ in an ensemble of mesoscopic metallic rings in a model of noninteracting electrons. The temperature dependence is explicit only in the Letter by Schmid. It seems appropriate to point out that an evaluation of Eqs. (12) and (13) of Ref. [1] shows the first harmonic in $2 e \Phi / h$ (where $\Phi$ is the threading flux) of this current to be significantly more sensitive to temperature than the interaction-induced current calculated by us $[4,5]$. The two dependences are shown in scaled form in Fig. 1.

The interaction-induced effect is well described by the interpolating formula $\exp \left[-T / 3 T_{1}\right]$. The one-electron contribution falls to $1 / e$ of its zero-temperature value at $0.7 T_{1}$. Here $T_{1}$ is $\hbar v_{F} l / 3 L^{2}$, with $v_{F}$ the Fermi velocity, $l$ the mean free path, and $L$ the perimeter of one ring.

For completeness we recall that the calculated $T=0$ amplitudes of the two contributions, in units of $e v_{F} / L$, are $I^{\text {s.e. }}=4 / A k_{F}^{2}$ (for vanishing phase breaking), where $A$ is the cross-sectional area and $k_{F}$ the Fermi momentum, and $I^{\text {int }}=\lambda^{*} 8 I / 3 \pi L$, where $\lambda^{*}$ is the effective coupling constant. It is also worth noting that strong spin-orbit scattering, which seems to be the relevant limit for the copper rings [6], does not affect $I^{\text {int }}$ [5], whereas the results of Ref. [7] imply a reduction of $I^{\text {s.e. }}$ by a factor of 4 .

The experimental result [6], in the above units, is given as $3 \times 10^{-3} \exp [-T /(80 \mathrm{mK})]$. The parameters recently quoted by Lévy [8] are $l=300 \AA, L=2.2 \times 10^{-4} \mathrm{~cm}$, and $A=350 \times 450 \AA^{2}$. The standard numbers for copper are $k_{F}=1.36 \AA^{-1}$ and $v_{F}=1.57 \times 10^{8} \mathrm{~cm} / \mathrm{sec}$.

Using the above numerical values, one obtains $3 T_{1}=75$ $\mathrm{mK}, I^{\text {s.e. }}=1.4 \times 10^{-5}$, and $I^{\text {int }}=1.2 \times 10^{-2} \lambda^{*}$. The calculation of $\lambda^{*}$ is difficult precisely because it is equivalent to calculating the transition temperature of a superconductor [9]. The value of 0.3 , estimated by us [4] from first-order perturbation theory in the screened Coulomb repulsion, gives good agreement with experiment, but it is known $[10,11]$ that there is a reduction due to multiple scattering in the Cooper channel. This correction is logarithmic [2,5], and we consider it extremely unlikely that it could amount to a factor of more than 5 to 10 . The open questions in this regard do not, however, diminish the force of the following conclusions: (i) The singleelectron effect is too small by almost 3 orders of magnitude, and too sensitive to temperature, to account for the observations. (ii) The temperature dependence of the interaction-induced effect is in very good agreement with the experiment, suggesting that some essential physics has been correctly identified, and, as a corollary, that this dependence should be calculated in future studies.

We thank Albert Schmid for stimulating discussions.

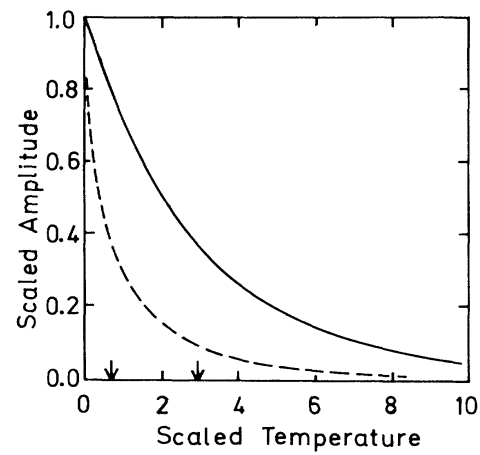

FIG. 1. Scaled amplitude vs temperature in units of $T_{1}$, defined in the text. Solid curve: interaction-induced current; dashed curve: one-electron contribution. The arrows indicate the temperature at which the amplitudes are reduced by $1 / e$, respectively.

This work was supported in part by the National Science Foundation under Grant No. DMR-88 15828.

Vinay Ambegaokar

Laboratory of Atomic and Solid State Physics Cornell University

Ithaca, New York 14850

\section{Ulrich Eckern}

Institut für Nukleare Festkörperphysik

Kernforschungszentrum Karlsruhe

Postfach 3640, W-7500 Karlsruhe 1,

Federal Republic of Germany

Received 1 March 1991

PACS numbers: $72.10 . \mathrm{Bg}, 05.30 . \mathrm{Fk}, 71.25 . \mathrm{Mg}, 75.20 . \mathrm{En}$

[1] A. Schmid, Phys. Rev. Lett. 66, 80 (1991); 66, 1379(E) (1991).

[2] F. v. Oppen and E. K. Riedel, Phys. Rev. Lett. 66, 84 (1991).

[3] B. L. Altshuler, Y. Gefen, and Y. Imry, Phys. Rev. Lett. 66, 88 (1991).

[4] V. Ambegaokar and U. Eckern, Phys. Rev. Lett. 65, 381 (1990).

[5] U. Eckern, Z. Phys. B 82, 393 (1991).

[6] L. P. Lévy et al., Phys. Rev. Lett. 64, 2074 (1990).

[7] B. L. Altshuler and B. I. Shlovskii, Zh. Eksp. Teor. Fiz. 91, 220 (1986) [Sov. Phys. JETP 64, 127 (1986).

[8] L. P. Lévy, Physica (Amsterdam) 169B, 245 (1991).

[9] V. Ambegaokar and U. Eckern, Europhys. Lett. 13, 733 (1990).

[10] B. L. Altshuler, D. E. Khmelnitskii, and B. Z. Spivak, Solid State Commun. 48, 841 (1983).

[11] B. L. Altshuler and A. G. Aronov, in Electron-Electron Interactions in Disordered Systems, edited by A. L. Efros and M. Pollak (North-Holland, Amsterdam, 1985), p. 1. 